\title{
Association of food quality and locations by cattle
}

\author{
DEREK W. BAILEY AND PHILLIP L. SIMS
}

Authors are assistant professor, Northern Agricultural Research Center, Montana State University, Havre, Mont. 59501; and range scientist and research leader, USDA-ARS, Woodward, Okla. 73801. At the time of the research, the senior author was ecologist, USDA-ARS, Woodward, Okla. 73801.

\section{Abstract}

Twelve yearling steers were observed in an 8-arm radial maze to determine the strength of the association between food quality and spatial locations following a 0 - or 30-day delay. The study was conducted using 3 qualities of feed, low (straw), medium (alfalfa pellets), and high (grain). During phase 1, all 8 arms contained dehydrated alfalfa pellets. In phase 2 , steers were fed either grain or wheat straw, in 2 arms (key arms). The remaining 6 arms contained alfalfa pellets. Six steers received straw in key arms, and 6 received grain. Key arms varied among steers and were selected so a change in arm selection patterns between phases would clearly be associated with corresponding changes in food quality. Straw was placed in arms that steers selected first during phase 1 , and grain was placed in arms that were selected last in phase 1 . Phase 3 began after a 0 - or 30-day delay following phase 2. In this phase, all arms contained alfalfa. Steers rarely reentered a previously entered arm indicating an accurate spatial memory for food location. The sequence of arm selections in phase 2 changed $(P<0.05)$ from the pattern established in phase 1 , which demonstrated that cattle can associate food quality with spatial locations. The delay between phase 2 and 3 did not affect $(P>0.05)$ the selection patterns of steers that had grain in key arms, but did appear to affect the number and sequence of arm entries for steers receiving low quality food in key arms during phase 2 . With no delay, steers that received straw in phase 2 did not enter key arms on the first day of phase 3, but after 30 days animals entered and consumed food in key arms. Steers with no delay entered key arms fewer $(P=0.03)$ times during phase 3 than steers that began 30 days later. This suggests that strength of the association between food quality and spatial locations can decline over time.

Key Words: forage quality, palatability, spatial memory, maze, cattle

Overgrazing and subsequent ecosystem degradation are often attributed to undesirable spatial distributions of livestock (Coughenour 1991). Managers often reduce stocking rates in an attempt to prevent uneven distribution and overgrazing in preferred areas (Holechek et al. 1989, Walker 1995). Rangeland managers must understand and be able to predict the spatial distribution of large herbivores to minimize the potential impacts of

This is published with approval of the Director of the Montana Agricultura Experiment Station Journal as Journal Series J-5059. The authors would like to thank Bill Cooper for his assistance in collecting behavioral observations.

Manuscript accepted 25 Feb. 1997. grazing on landscape processes. Fencing, water developments, riding, salting and other practices have been used to improve grazing distribution (Cook 1967, Bailey and Rittenhouse 1989). However, the underlying behavior mechanisms and processes that result in large herbivore grazing patterns must be understood before innovative techniques can be developed to improve grazing distribution (Bailey et al. 1996).

Large herbivores generally allocate time spent in a feeding area or plant community in proportion to available resources (Senft et al. 1987, Pinchak et al. 1991). Bailey et al. (1989a, 1989b) suggested that spatial grazing patterns of cattle and other large herbivores may result from animals returning to nutrient-rich areas more frequently than nutrient-poor areas. This behavioral mechanism assumes that herbivores have accurate spatial memories for both the quantity and quality of food encountered (Bailey et al. 1996). Empirical studies (Bailey et al. 1989a, Laca 1995) have shown that cattle can remember food locations. Bailey et al. (1989b) demonstrated that cattle associate spatial locations with food quantity, yet it is not clear if cattle use memory to associate food quality with spatial locations.

Bailey et al. (1996) developed a conceptual model to predict the distribution of large herbivores based on spatial memory and frequency of feeding site selection. The model assumes that animals remember good and poor feeding sites based on quantities and/or quality of forages and return most frequently to good sites. The model specifically predicts periodic sampling of all feeding sites. It predicts that herbivores will avoid nutrient-poor feeding sites after their initial visit, but eventually animals will return to poor sites and reevaluate forage conditions. The basis for periodic sampling is the model's assumption that the influence of previous foraging experiences on subsequent feeding site selections declines over time. Memories of previous foraging experiences in the model decay, and eventually the simulated herbivore forgets how bad or how good a feeding site was. Memory decay can also be considered as a change in information value (Devenport and Devenport 1993, 1994) over time. After long delays, previous foraging experiences provide less information and should not be weighted as heavily in feeding site selection decisions because forage conditions change.

The primary objective of this study was to determine if cattle could associate spatial locations with the quality of food found there. We hypothesized that cattle would quickly accomplish this memory task and that selection of feeding sites would be based on food quality. A second objective was to determine if the strength of such associations, if any, would decline over time. Our hypothesis was that any preferences for spatial locations resulting from associations with food quality would be more pronounced if the animal was evaluated the next day than if animals were evaluated 30 days later. 


\section{Methods}

\section{Study Apparatus}

The study was conducted in an 8-arm radial maze similar to that used by Bailey et al. (1989a). Two strands of electric fence were used for the perimeter of the maze. The maze consisted of 8 arms that were $3 \mathrm{~m}$ wide and $20 \mathrm{~m}$ in length and a center decision area that was $20 \mathrm{~m}$ in diameter (Fig. 1). An opaque feeder $(0.5 \mathrm{~m}$ diameter rubber tub) was placed at the end of each arm. The maze was constructed in a native rangeland pasture with a few trees and shrubs located outside but, near the maze. A holding pen and release pen were constructed near the maze. The lane from the release pen and the gate into the maze were also constructed from electric fence. A visual barrier was constructed on the 3 sides of the release pen facing the maze (Fig. 1) to prevent animals from observing the maze.

\section{Experimental Approach}

Overview. Three yearling-crossbred (Bos taurus $\times$ Bos indicus) steers weighing approximately $250 \mathrm{~kg}$ were randomly assigned to each of 4 groups. For the first 5 days, all cattle were treated the same. During the next 7 days (days 6 to 12), the quality of available food was changed for all cattle in $25 \%$ of the feeding locations. For 2 groups, quality of available food improved, and a higher quality food was provided in $25 \%$ of the locations. Food quality declined for the other 2 groups in $25 \%$ of the feeding locations. The focus of the study was the feeding locations, termed key arms, where food type was changed during days 6 to 12. We observed if steers preferred or avoided key arms after the

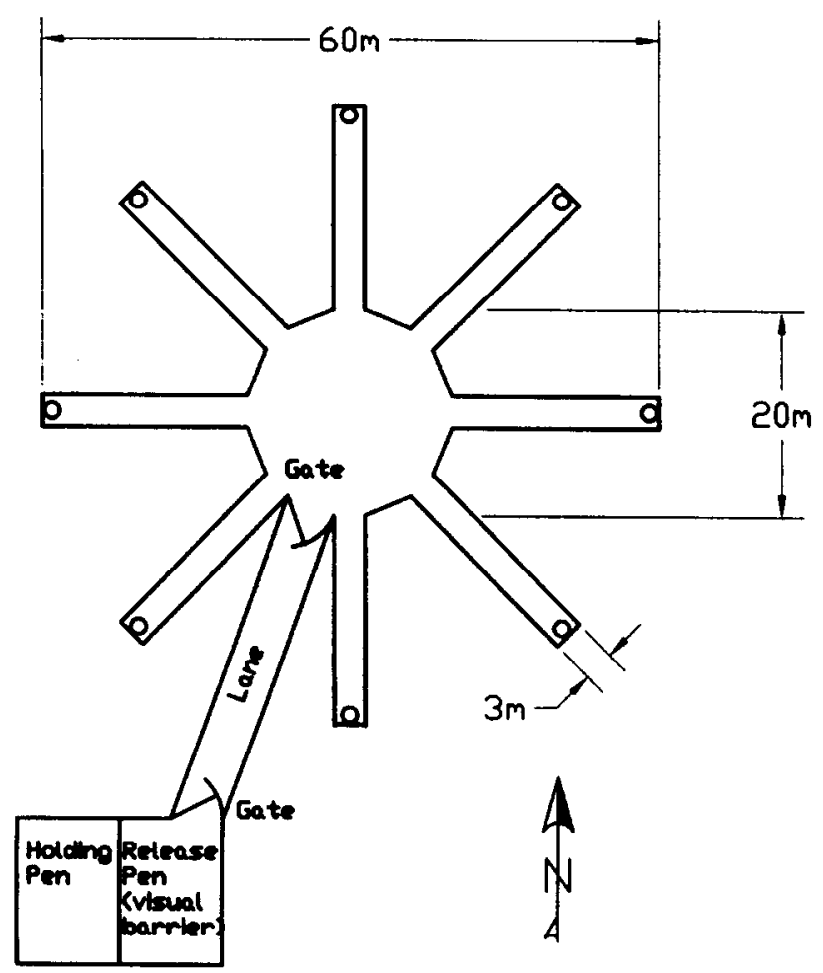

Fig. 1. Diagram of 8-arm radial maze with the holding pen and release pen with a visual barrier on the radial maze sides. food was changed. Following this exposure, all groups were reevaluated for an additional 5 days with all feeding locations containing the same food, similar to days 1 to 5. Two groups (food quality increased and decreased) were reevaluated on days 13 to 17 , and the other 2 groups were reevaluated 30 days later on days 42 to 46 . The purpose of this reevaluation was to determine if feeding site selection patterns persisted.

Feed description. Before the study a variety of grains, forages and other foodstuffs were fed to steers to identify highly palatable (high quality), moderately palatable (moderate quality) and a relatively unpalatable (low quality) foodstuffs. A grain mix (grain) was the most palatable and was selected as the high quality food (Table 1). Dehydrated alfalfa pellets (alfalfa) were less palatable than the grain mix and were selected as the moderate quality foodstuff, and wheat straw (straw) was virtually unpalatable and was selected as the low-quality foodstuff. Table 1 lists the crude protein content and in vitro organic matter disappearance (IVOMD) of the 3 feeds which were determined from a semimicro-Kjeldahl method (Bremner and Breitenbeck 1983) and a Tilley and Terry 2-stage technique (Tilley and Terry 1963) as modified by White et al. (1981).

Table 1. Chemical analyses and categorizations of foodstuffs placed at the end of arms in a radial maze used to evaluate memory.

\begin{tabular}{lllcc}
\hline \hline Feed & Palatability & Quality & $\begin{array}{c}\text { Crude } \\
\text { protein }\end{array}$ & $\begin{array}{c}\text { In vitro OM } \\
\text { disappearance }\end{array}$ \\
\hline & & & $-\ldots-1 \%)-\ldots--$ \\
Grain mix & High & High & 14.1 & 82.7 \\
Dehydrated alfalfa pellets & Moderate & Moderate & 19.0 & 59.9 \\
Wheat straw & Low & Low & 2.4 & 41.4 \\
\hline
\end{tabular}

'Grain mix consisted of corn, milo, barley, oats, molasses and a cottonseed meal protein supplement.

Phase 1. As indicated above, the study was conducted in 3 phases. In the first phase (days 1 to 5), cattle were trained to expect the same quality and quantity of food in all maze arms. Feeders in all 8 arms contained $0.1 \mathrm{~kg}$ of alfalfa. Steers were individually observed in the maze, and arm selection patterns were documented. Cattle may prefer 1 maze arm over others because of handedness and other factors (Hosoi et al. 1995).

Definition of Key Arms. For 2 of the 4 groups, the 2 arms that were most frequently chosen last during phase 1 were identified and termed key arms. For the other 2 groups, the 2 arms most frequently chosen first during phase 1 were identified and termed key arms. The only restriction in selecting key arms was that key arms were never adjacent. At least 1 arm separated key arms to ensure key arms were 2 distinct locations and not just a region of the maze. Since the selection of key arms was based on individual animal arm selection patterns, key arms varied among steers and within treatment groups. Key arms assigned to a steer did not change during the study.

Phase 2. During phase 2 (days 6 to 12), the 2 key arms contained either grain or straw $(0.1 \mathrm{~kg})$ and the 6 other arms contained alfalfa $(0.1 \mathrm{~kg})$. Grain was placed in key arms that were most frequently chosen last in phase 1 ( 2 groups). For the other 2 groups (key arms choosen first), the key arms contained straw. In other words, grain was placed in arms that were avoided in phase 1 , and straw was placed in arms that were preferred. The purpose of the second phase was to determine if cattle would associate a 
different food quality with specific locations (maze arms). A change in the arm selection pattern from phase 1 to phase 2 should indicate that cattle associated food quality with spatial locations. The rationale for selecting the least-preferred arms for grain and the most-preferred arms for straw was to increase the rigor of the study. Changes in maze arm selection patterns between phases 1 and 2 could be more readily attributed to food quality than to non-food factors such as turning tendency or handedness (Ganskopp 1995, Hosoi et al. 1995). The animal's preference or avoidance of an arm because of the associated food quality in phase 2 had to overcome its initial avoidance or preference of that arm in phase 1 which resulted from non-food factors.
Phase 3. Phase 3 was a reevaluation of arm selection patterns with all arms containing $0.1 \mathrm{~kg}$ of alfalfa. This phase lasted 5 days, and the starting date was either 0 or 30 days from the end of phase 2 depending on the steer group. Phase 3 began the day after phase 2 for one of the groups receiving grain in phase 2 (GRAIN0) and one of the groups receiving straw (STRAW-0). The starting date began 30 days after phase 2 for the other group receiving grain (GRAIN-30) and straw (STRAW-30). The purpose of the third phase was to evaluate if any selection patterns developed in phase 2 persisted. Figure 2 summarizes the differences among the 4 treatment groups.

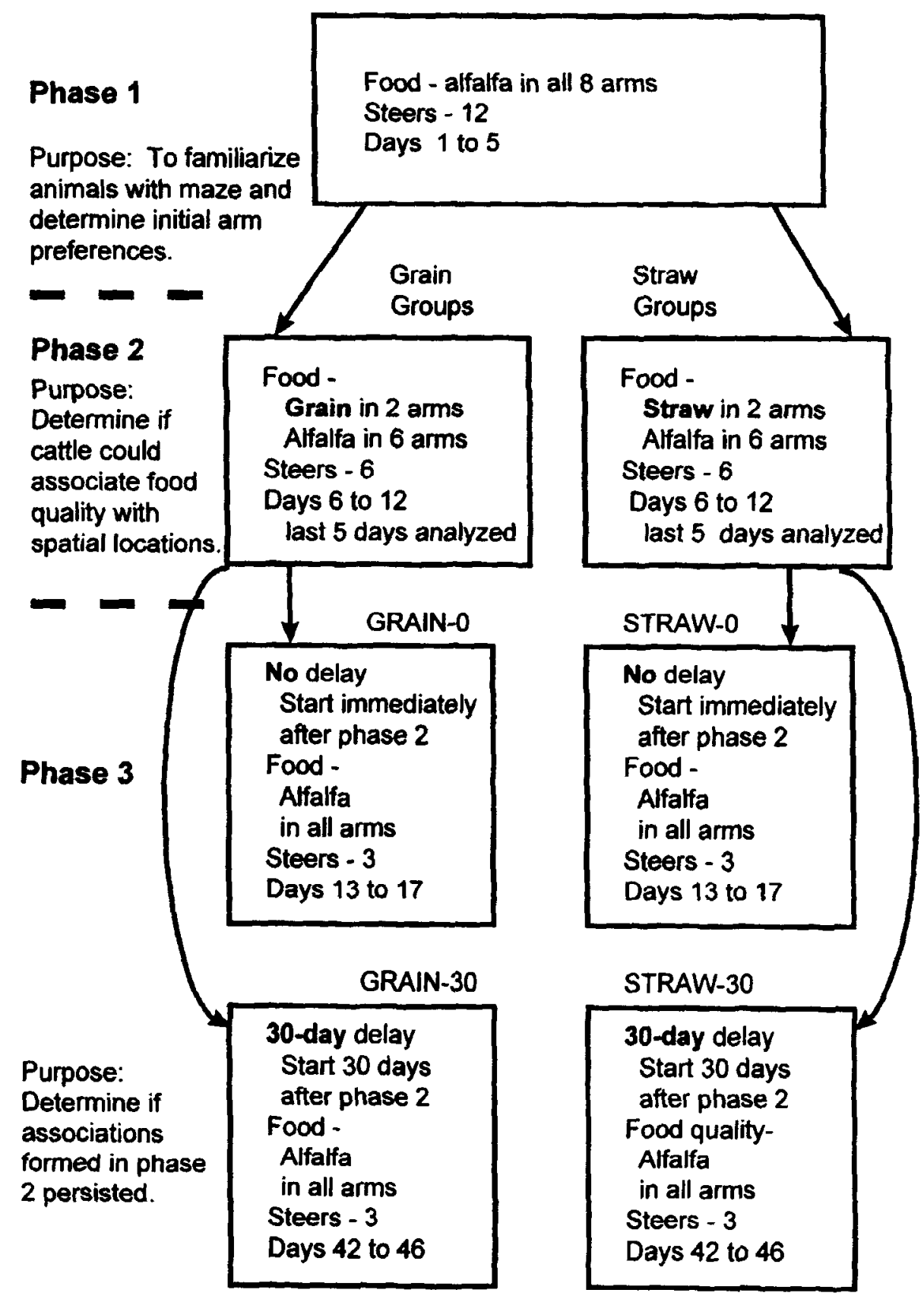

Fig. 2. Flow chart describing the design of the study conducted in an 8-arm radial maze. At the beginning and end of the study, dehydrated alfalfa pellets (alfalfa) was placed at the end of all arms, while alfalfa and either a grain mix (grain) or wheat straw (straw) was fed in 2 of the 8 arms (key arms). 


\section{Protocol}

The 2 treatment groups with a 30-day delay between phases 2 and 3 (GRAIN-30 and STRAW-30) were trained and observed during the same time periods, (phases 1 and 2, 16 March 1995 to 10 April 1995, and phase 3, 10 May 1995 to 16 May 1995). The other 2 groups (GRAIN-0 and STRAW-0) were observed from 14 April 1995 to 9 May 1995. Six steers (2 treatment groups) were individually observed once each day beginning about 0800 hours. Observations usually ended by 1500 hours. When steers were not in the maze or in the holding pen waiting to be observed, they grazed in an adjacent native rangeland pasture. Steers were gathered and placed in the holding pen about $30 \mathrm{~min}$ before observations began. During the periods that steers were not observed (e.g., 30-day delay period), they also grazed in a similar native rangeland pasture located nearby. Thus, diets of all steers should have been similar during the study except for the consumption of food in the maze. However, the amount of food consumed in the maze ( $0.8 \mathrm{~kg} /$ day) accounted for less than $16 \%$ of their total daily intake assuming the steers consumed at least $5 \mathrm{~kg} /$ day, i.e., $2 \%$ of their body weight (NRC 1996).

Before entering the maze, steers were placed in the release pen while the observer reloaded feed at the end of each arm. The observer herded the steer from the release pen to the gate leading into the central decision area of the maze, and observed its activities from a location outside the maze near the holding pen. The observer recorded the sequence that arms were entered and whether food was consumed. Steers were removed from the maze after they entered all arms containing alfalfa or grain in phases 1 and 2. In phase 3, steers in the STRAW-0 and STRAW-30 groups were allowed to leave after entering the 6 arms containing alfalfa in phase 2. Steers in the GRAIN-0 and GRAIN-30 groups were allowed to leave the maze during phase 3 after they entered all 8 arms or after they entered the same arm more than 2 times. After the animal left the maze, it was placed in the holding pen. Another steer was placed in the release pen and the process was repeated for each steer. The sequence in which steers were observed during a day was randomized.

Animals were assisted by the observer into unentered arms after 30 minutes or after a third reentry of any arm during phases 1 and 2. Anytime the observer was within $5 \mathrm{~m}$ of the decision area the animal's subsequent choice was recorded as assisted. Assists were generally the last arm entered during a trial, and usually required direct herding. Steers were approached from the arm end they were in and herded to the decision area and into the unentered arm. During phase 3, steers were not assisted.

Periodically in all phases, steers would graze in an arm after consuming the food. When this occurred, the observer would attempt to start the animal moving toward the decision area without influencing its subsequent arm choice. The observer would approach the animal from the arm end and move toward the animal by walking in the center of the arm. Once the animal started moving, the observer slowly backed toward the arm end and then left the maze. If the observer remained at least $5 \mathrm{~m}$ from the decision area while starting the animal moving, the event was recorded as push. If the observer was required to be within $5 \mathrm{~m}$ of the decision area, the choice was considered as an assist. The criteria used to differentiate between assists and pushes are more stringent than those used in other maze studies with cattle (Bailey et al., 1989a, $1989 \mathrm{~b}$ ). It is unlikely that the observer affected animal movement in the decision area after a push since $5 \mathrm{~m}$ is usually outside a steer's flight zone, especially steers accustomed to the observer (Grandin 1989). The arms were relatively narrow $(3 \mathrm{~m})$, and steers generally followed trails down the center of the arm to avoid the electrified wires. Thus, the observer would be unlikely to influence the tendency of steers to turn while they were in the arm.

\section{Description of Dependent Variables}

Most of the dependent variables used in this study involves number of choices. If a steer traveled more than $4 \mathrm{~m}$ into the arm before turning around or backing out of the arm, this behavior was considered an arm entry and a choice. If a steer entered an arm but traveled less than $4 \mathrm{~m}$ down the arm before leaving, this behavior was not considered an arm entry or a choice, and it was not used in the analysis.

Spatial memory of food within the maze was evaluated using the number of correct choices in the first 8 entrances, similar to other maze studies (Olton 1978, Bailey et al. 1989a, 1989b). A choice was considered correct if a steer entered a previously unentered arm without assistance from the observer. If a steer entered a previously entered arm, a repeat, the choice was considered incorrect. The choice was also considered incorrect if the observer herded the steer into an arm, an assist. The choice after a push could be either correct or incorrect since it was not considered an assist, and the observer avoided influencing the animal's decision. In an 8 -arm maze, 8 correct choices in 8 entrances occurs when animals make no mistakes (perfect performance) while 5.3 correct choices in 8 entrances is the value expected by chance (Olton 1978). In cases when steers did not enter 8 arms during an observation period, there was no adjustment. For example, 6 correct choices in 8 entrances was recorded if a steer entered 6 previously unentered arms without assistance during an observation period.

The number of choices before $1 \mathrm{key}$ arm was selected and the number of choices before both key arms was selected during an observation period were used to evaluate if the pattern of arm selections changed during the study. This allowed us to document if key arms were selected first or last during an observation period. For example, a value of 6 would be recorded if the first key arm entered during an observation period was the steer's sixth choice. Although key arms were identified at the end of phase 1, the numbers of choices to these arms during phase 1 were used in the analyses to evaluate if arm selection patterns in phases 1 and 2 differed. When 1 or 2 key arms were avoided during an observation period, the number of choices to 1 or 2 arms was calculated by increasing the total number of choices by 1 or 2 , respectively.

\section{Statistical Analyses}

Analysis of variance was used to compare results from the treatment groups across the 3 phases of the study (SAS 1988). The 5 values from each steer during phases 1 and 3 were pooled for analyses of variance, and for phase 2 only the values from the last 5 days were pooled and used. For analyses of the number of correct choices in the first 8 entrances and pushes, the model included food quality (grain vs. straw in phase 2), delay interval between phase 2 and 3 ( 0 vs. 30 day delay), phase, quality by delay interval interaction, and steer. The between steer variance component was used as the error term. To analyze the number of choices to 1 or $2 \mathrm{key}$ arms, groups receiving grain in phase 2 (GRAIN-0 and GRAIN-30) were analyzed separately from 
groups that received straw (STRAW-0 and STRAW-30) because the change in values from one phase to another was expected to he in opposite directions for these groups. The model included delay interval, phase, steer within delay interval and the phase by delay interval interaction. The between steer variation was used as the error term.

T-tests ( $4 \mathrm{df}$ ) were used to compare the 0 - and 30-day groups (GRAIN-0 vs. GRAIN-30 and STRAW-0 and STRAW-30) for the number of choices to 1 key arm in phase 3 , number of key arms not entered in phase 3 (pooled data) and the change in the number of choices from the last day of phase 2 to the first day of phase 3 . One-sided t-tests were used because the differences between the long and short delays were expected in only one direction.

The number of choices to 1 key arm on the last day of phase 2 and the first day of phase 3 were compared using paired t-tests. Separate analyses were completed for each of the 4 groups, and steer was used to pair values ( $2 \mathrm{df}$ ).

\section{Results and Discussion}

\section{Evaluation of Spatial Memory}

During phase 1, the mean number of correct choices in the first 8 entrances was 7.0 for all groups, which is greater $(P<0.05)$ than the 5.3 value expected by chance (Olton 1978). This level of performance in an 8-arm radial maze indicates an accurate spatial memory, but it is slightly less than the accuracy ( 7.6 to 7.8 correct choices in the first 8 entrances) reported for cattle by Bailey et al. (1989a). The number of correct choices in the first 8 entrances was similar $(P>0.25)$ throughout the study (Table 2$)$. Changing food quality in some of the feeding sites did not result in steers selecting previously entered arms, repeats, or increase the need of assistance from the observer.

Steers often grazed during the study, and the observer had to start them moving with a "push" (Table 2). The number of pushes remained consistent $(\mathrm{P}>0.2)$ across all phases, and there were no differences $(P>0.2)$ between the groups.

Table 2. Evaluation of spatial memory of steers in an 8-arm radial maze during the 3 phases of the study. Means were from groups that received grain (GRAIN-0 and GRAIN-30) and straw (STRAW-0 and STRAW-30) during phase 2.

\begin{tabular}{lccc}
\hline Group & Phase & $\begin{array}{c}\text { Correct } \\
\text { choices in } 8 \\
\text { entrances }^{1}\end{array}$ & Pushes $^{2}$ \\
\hline Grain & 1 & 7.0 & 1.9 \\
& 2 & 7.6 & 1.1 \\
Straw & 3 & 7.6 & 1.0 \\
& 1 & 7.0 & 2.4 \\
& 2 & 6.7 & 2.3 \\
\hline
\end{tabular}

${ }^{1}$ Correct choices were defined by steers entering previously unentered arms (during an observation period) without the observer herding the animal into an arm. A value of 8 indicates that as steers entered all 8 arms without assistance from the observer and did not reenter any arms, perfect performance. A value of 5.3 is expected by chance (Olton 1978).

${ }^{2}$ Pushes occurred when the observer started the animal moving out of a maze arm, but avoided influencing the steer's next choice. Choices following a push could be correct or incorrect.

\section{Association of Food Quality and Locations}

The mean number of choices before 1 key arm was selected in phase 2 was lower $(P=0.04)$ than phase 1 for the GRAIN-0 and GRAIN-30 groups (Table 3). The number of choices before both key arms were selected was also lower $(P=0.11)$ in phase 2 than phase 1 for these 2 groups. Steers selected key arms containing grain during their first few arm choices in phase 2, instead of selecting them last as they did in phase 1 .

For the STRAW-0 and STRAW-30 groups, the mean number of choices before 1 key arm was selected was greater $(P<0.05)$ in phase 2 than in phase 1 (Table 3 ). The number of choices

Table 3. The mean number of choices before 1 or 2 identified arms (key arms) were selected in an 8-arm radial maze.

\begin{tabular}{lccc}
\hline \hline Groups & Phase & $\begin{array}{c}\text { Choices to } \\
1 \text { key arm }^{3}\end{array}$ & $\begin{array}{c}\text { Choices to } \\
2 \text { key arms }\end{array}$ \\
\hline Grain $^{2}$ & 1 & $4.3 \mathrm{a}$ & $7.7 \mathrm{a}$ \\
& 2 & $2.7 \mathrm{~b}$ & $5.9 \mathrm{~b}$ \\
& 3 & $3.1 \mathrm{ab}$ & $6.7 \mathrm{ab}$ \\
Straw $^{3}$ & 1 & $1.1 \mathrm{a}$ & $5.0 \mathrm{a}$ \\
& 2 & $4.4 \mathrm{~b}$ & $7.5 \mathrm{~b}$ \\
& 3 & $4.6 \mathrm{~b}$ & $7.1 \mathrm{~b}$ \\
\hline
\end{tabular}

${ }^{\mathrm{I}}$ Choices refer to arm entrances in a 8-arm radial maze. Key arms were identified for each steer using arm selection patterns from the first 5 days of the study, phase 1 .

${ }^{2}$ Means are pooled data from the GRAIN-0 and GRAIN-30 groups (see text). Key arms in these groups were selected last during phase 1.

These groups received grain in key arms during phase 2

${ }^{3}$ Means are pooled data from to the STRAW-0 and STRAW-30 groups (see text). Key arms in these groups were selected first in phase 1.

These groups received straw in key arms during phase 2 .

a,b Values within a column and group (ie., grain and straw) followed by different letters differ $(P<0.05)$.

before both key arms was selected was also greater $(P=0.04)$ in phase 2 than phase 1 . Steers clearly avoided key arms containing straw in phase 2 even though they selected these arms first during phase 1. In most cases during the later part of phase 2, steers did not enter arms that contained straw (Fig. 3). After consuming the alfalfa, 6 arms or 6 correct choices, they went to the exit gate and waited for it to be opened.

The change in arm selection patterns from phase 1 to phase 2 demonstrates that cattle can associate food quality or palatability with a spatial location. These results agree with those observed by Scott et al. (1995). In that study, lambs changed their selection of feeding sites when placement of a desired food and an undesired food were switched.

In this study, it is not possible to distinguish whether cattle associated spatial locations with food quality or with food palatability. Food quality and food palatability are typically confounded. Provenza (1995) suggests that palatability is a function of food quality through post-ingestive feedback. In any case, steers remembered locations that contained preferred foods and selected them first, and they remembered locations that contained the relatively unpalatable food (straw) and selected them last or avoided them altogether.

\section{Persistence of Associations}

Food Quality Effects. The mean number of choices before 1 key arm was selected in phase 3 was similar $(P<0.05)$ to those in phases 1 and 2 for the groups that received grain (Table 3 ), GRAIN-0 and GRAIN-30. However, the choices to 1 key arm were greater $(\mathrm{P}=0.04)$ in phase 3 than phase 1 for the groups that 
received straw, STRAW-0 and STRAW-30. When food quality declines in a location from a high level to a low level, herbivores quickly observe the change because they frequently return to nutrient rich locations (Bailey 1995). However, an improvement in food quality from a low to high level may not be recognized immediately because herbivores may avoid locations with low food quality. Steers in this study, quickly learned that food in key arms changed from grain to alfalfa in phase 3 because they did not avoid key arms.

Grain Groups. The GRAIN-0 and GRAIN-30 groups did not differ $(P>0.05)$ in the mean number of choices to 1 or 2 key arms when evaluated across the study. During phase 3 , the mean number of choices to 1 key arm was also similar $(P>0.25)$ for the GRAIN-0 (3.6) and GRAIN-30 (2.7) groups. The mean number of choices to 1 key arm on the last day of phase 2 was similar (P $>0.21$ ) to the first day of phase 3 for the GRAIN-0 and GRAIN-30 groups (separate paired-t tests). For steers that received grain in phase 2, delaying the start of phase 3 by 30 days had little effect on arm selection patterns.

Straw Groups. The STRAW-0 and STRAW-30 groups did not differ $(P>0.15)$ in the mean number of choices to 1 or 2 key arms when evaluated across the study. During phase 3 , the mean number of choices to 1 key arm was 5.7 for the STRAW-0 group and 3.6 for the STRAW-30 group, but the difference was not significant $(P=0.14)$. The mean number of choices to 1 key arm from the last day of phase 2 to the first day of phase 3 increased ( $P=0.05$ ) for the STRAW-30 group, but the STRAW-0 group did not change $(P>0.25)$. The change in the mean number of choices from the last day of phase 2 to the first day of phase 3 was 2.7 for the STRAW-30 group which was greater $(P=0.05)$ than the change of -1.0 for the STRAW-0 group. This suggests that the association between the low quality foods and spatial locations declined during the 30-day interval.

The most convincing evidence, however, is that none of the steers from the STRAW-0 group entered a key arm on the first day of phase 3, and all of the steers from the STRAW-30 group entered both key arms and consumed all of the feed (Fig. 3). During the 5 days of phase 3, steers in STRAW-0 group entered key arms fewer $(P=0.03)$ times than the STRAW-30 group. Steer 310 in the STRAW-0 group did not enter a key arm during all of phase 3, and steer 409 in that group only entered a key arm twice. These steers avoided key arms more strongly in phase 3 than in phase 2. However, the other steer (357) in the STRAW-0 group behaved differently than the other steers for most of phase 3 . On the first day of phase 3 , steer 357 , like the other 2 steers, avoided and did not enter the key arms, but on the second day of phase 3 steer 357 entered a key arm on the second choice and later entered the second key arm. Steer 357 entered both key arms for the remainder of phase 3 (Fig. 3). The reason that steer 357 behaved differently than the other steers in the group is not known, but perhaps the steer was purposely sampling.

During phase 3, steers in the STRAW-0 group avoided key arms even though they contained alfalfa. This suggests that cattle used memory rather than visual or olfactory cues to locate alfalfa. Bailey et al. (1989a) concluded that cattle did not use olfactory cues to locate food in radial- and parallel-arm mazes. Feeders used in maze studies are opaque which prevent animals for using visual cues. Animals apparently can associate features of a spatial location (visual and other attributes) with the quality and quantity of food found there (Bailey et al. 1996).
STRAW-0 Group

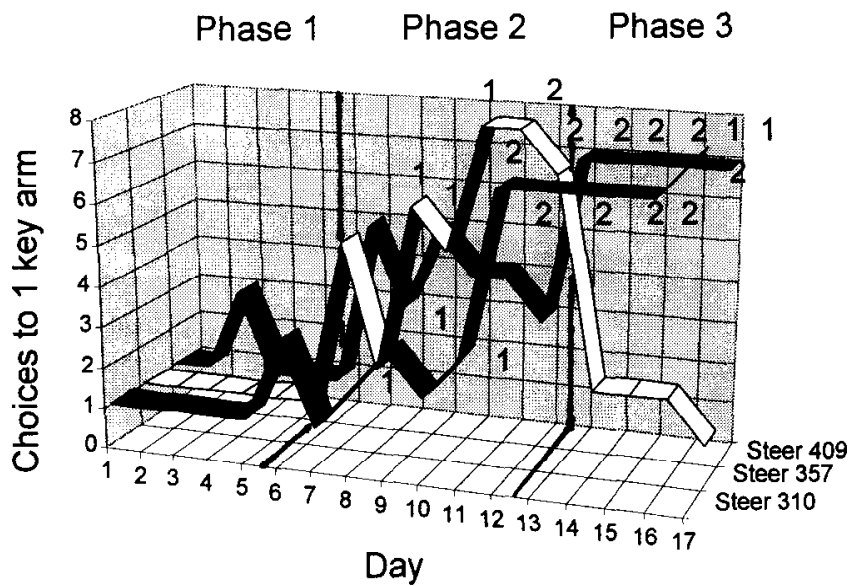

STRAW-30 Group

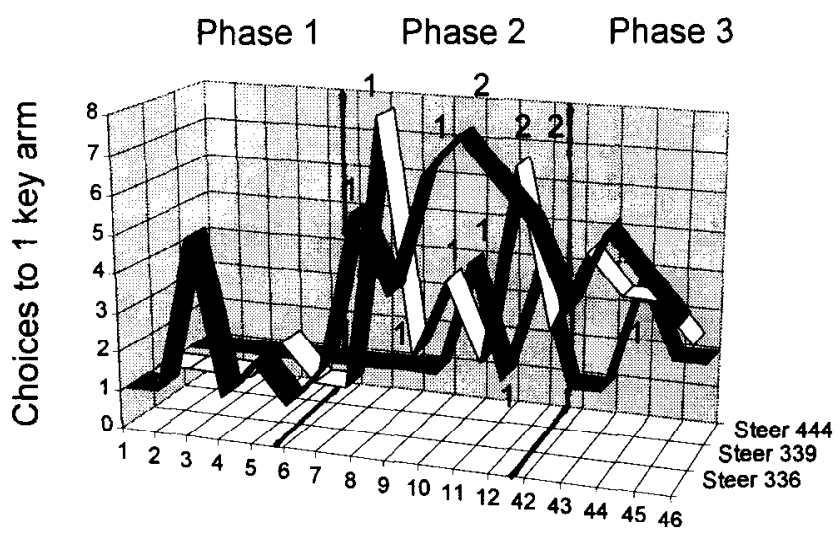

Day

Fig. 3. Number of choices before 1 of the 2 identified feeding sites (key arms) was selerted each day for individual animals in the 2 groups receiving straw in phase 2, STRAW-0 (no delay between phases 2 and 3 ) and STRAW-30 (30-day delay). Key arms for these groups were identified because arm selection patterns in the radial maze during phase 1 indicated that steers selected them first. Straw was placed in the 2 key arms during phase 2 . The numbers " 1 " and " 2 " on the graph indicate that the steer did not enter 1 or 2 key arms, respectively, on that day of the study.

\section{Application to Grazing Models}

The conceptual grazing model developed by Bailey et al. (1996) assumes that cattle can associate forage quality and quantity with spatial locations and that the strength of these associations may weaken or decay over time. The premise of this model is that cattle remember areas and return to areas with greater resources (higher forage quality and/or quantity) and usually avoid areas with fewer resources. However, the model predicts that areas with fewer resources are periodically visited because animals tend to forget how poor the area was (memory decay) or because the reliability of the information from previous foraging experiences declines over time (Devenport and Devenport 1993, 1994). Bailey et al. (1989b) demonstrated that cattle can associate food quantity with spatial locations. This study shows that cattle 
can remember the quality (or palatability) found at different spatial locations. This study also suggests that the strength of the association between food quality (or palatability) and spatial locations declines over time.

\section{Implications}

This study supports the conceptual model described by Bailey et al. (1996) that cattle memory of feeding sites with low-quality foods declines over time. Memory decay may be an important mechanism for explaining grazing movements of cattle. Further research is needed to determine if memory decay is the reason cattle eventually return to feeding sites with fewer resources. Alternatively, animals may purposely sample areas in their home range on a periodic basis to reassess available food resources. Larger-scale field studies are needed to determine if the results from this study are applicable to livestock and other large herbivores grazing rangelands.

\section{Literature Cited}

Bailey, D.W. 1995. Daily selection of feeding areas by cattle in homogeneous and heterogeneous environments. Appl. Anim. Behav. Sci. 45:183-199.

Bailey, D.W. and L.R. Rittenhouse. 1989. Management of cattle distribution. Rangclands 11:159-161.

Bailey, D.W., L.R. Rittenhouse, R.H. Hart, and R.W. Richards. 1989a. Characteristics of spatial memory in cattle. Appl. Anim. Behav Sci. 23:331-340.

Bailey, D.W., L.R. Rittenhouse, R.H. Hart, D.M. Swift, and R.W. Richards. $1989 \mathrm{~b}$. Association of relative food availabilities and locations by cattle. J. Range Manage. 42:480-482.

Bailey, D.W., J.E. Gross, E.A. Laca, L.R. Rittenhouse, M.B. Coughenour, D.M. Swift, and P.L. Sims. 1996. Mechanisms that result in large herbivore grazing distribution patterns. J. Range Manage. 49:386-400.

Bremner, J.M. and G.A. Breitenbeck. 1983. A simple method for determination of ammonium in semimicro-Kjeldahl analysis of soils and plant materials using a block digester. Commun. In: Soil Sci. Plant Anal. 14:905-913.

Cook, C.W. 1967. Increased capacity through better distribution on mountain ranges. Utah Sci. 28:39-42.

Coughenour, M.B. 1991. Spatial components of plant-herbivore interactions in pastoral, ranching and native ungulate ecosystems. J. Range Manage. 44:530-542.

Devenport, J.A. and L.D. Devenport. 1993. Time-dependent decisions in dogs (Canis familiaris). J. Comparative Psychology 107:169-173.

Devenport, L.D. and J.A. Devenport. 1994. Time-dependent averaging of foraging information in least chipmunks and golden-mantled squirrels. Anim. Behav. 47:787-802.

Ganskopp, D. 1995. Free-ranging angora goats: left- or right-handed tendencies while grazing? Appl. Anim. Behav. Sci. 43:141-146.

Grandin, T. 1989. Behavioral principles of livestock handling. Professional Anim. Sci. 5(2):1-11.

Holechek, J.L., R.D. Peiper, and C.H. Gerbe. 1989. Range management principles and practices. Prentice Hall. Englewood Cliffs, N.J.

Hosoi, E., L.R. Rittenhouse, D.M. Swift, and R.W. Richards. 1995. Foraging strategies of cattle in a Y-maze: influence of food availability. Appl. Anim. Behav. Sci. 43:189-196.

Laca, E.A. 1995. Spatial memory and foraging efficiency of cattle Abstr., 48th Anu. Meeting. Soc. Range Manage., 1995, Phoenix, Ariz.

NRC. 1996. Nutrient requirements of beef cattle. 7th Ed. Nat. Acad. Sci., Washington, D.C.
Olton, D.S. 1978. Characteristics of spatial memory, p. 341-373. In: S.A. Hulse, H. Fowler, and W.K. Honig (eds.). Cognitive processes in animal behavior. Erlbaum, Hillsdale, N.J.

Pinchak, W.E., M.A. Smith, R.H. Hart, and J.W. Waggoner, Jr. 1991. Beef cattle distribution patterns on foothill range. J. Range Manage, 44:267-275.

Provenza, F.D. 1995. Postingestive feedback as an elementary determinant of food selection and intake in ruminants. J. Range Manage. 48:2-17.

SAS. 1985. User's Guide: Statistics, Version 5 Edition. SAS Inst. Inc., Cary, N.C.

Scott, C.B., F.D. Provenza, and R.E. Banner. 1995. Dietary habits and social interactions affect choice of feeding location by sheep. Appl. Anim. Behav. Sci. 45:225-237.

Senft, R.L., M.B. Coughenour, D.W. Bailey, L.R. Rittenhouse, O.E. Sala, and D.M. Swift. 1987. Large herbivore foraging and ecological hierarchies. BioScience 37:789-799.

Tilley, J.M.A. and R.A. Terry. 1963. A two-stage technique for the in vitro digestion of forage crops. J. Brit. Grassl. Soc. 18:104-111.

Walker, J.W. 1995. Viewpoint: grazing management and research now and in the next millennium. J. Range Manage. 48:350-357.

White, L.M., G.P. Hartman, and J.W. Bergman. 1981. In vitro digestibility, crude protein, and phosphorus content of straw of winter wheat, spring wheat, barley, and oat cultivars in eastern Montana. Agron. J. 73:117-121. 